\title{
Molecular biomarker profile of EGFR copy number, KRAS and BRAF mutations in colorectal carcinoma
}

\author{
Rong Rong, Jamie Tull, Shengle Zhang \\ Department of Pathology, SUNY Upstate University, Syracuse, NY, USA
}

Correspondence: Shengle Zhang. Address: Department of Pathology, SUNY Upstate Medical University, Syracuse, NY, USA. Telephone: 315-464-7131. Fax: 315-464-7130. E-mail: zhangs@upstate.edu

Received: April 25, 2012

DOI : $10.5430 /$ jst.v2n4p27

\author{
Accepted: July 9, 2012 \\ Published: August 1, 2012 \\ URL: http://dx.doi.org/10.5430/jst.v2n4p27
}

\begin{abstract}
Anti-EGFR therapy is approved by US Food and Drug Administration (FDA) as mono-therapy or as part of combination chemotherapy for metastatic colorectal carcinoma. Monoclonal anti-EGFR antibodies cetuximab and panitumumab have shown benefits for those patients with wild-type KRAS gene. Patients with KRAS mutation in codon 12 or 13 are unresponsive to targeted anti-EGFR therapy. Mutations of BRAF, the main downstream target gene of KRAS, also negatively impact the patients' response to the therapy. On the contrary, EGFR gene amplification in colon cancer predicts a better response to anti-EGFR therapy. The correlation of EGFR amplification with KRAS and BRAF mutation status is not only of academic interests but also of clinical importance. Previous studies have shown that EGFR mutation is mutually exclusive with KRAS and BRAF mutations in lung cancer. However, there is limited data on colorectal cancer so far. In this study, 28 colorectal cancer samples are tested for KRAS and BRAF mutations by PCR and for EGFR gene copy number by fluorescence in situ hybridization (FISH). EGFR high-copies, KRAS mutation and BRAF mutation are found in 15 (54\%), 13 (46\%) and 3 (11\%) samples, respectively. A mutually-exclusive pattern is seen between KRAS mutations (13 positive samples) and BRAF mutations (3 positive samples). However, high EGFR high copy number is not "mutually-exclusive" with KRAS or BRAF mutations. Six samples with KRAS mutations and 2 with BRAF mutation show "co-existing" high EGFR copy number. These account for $29 \%$ of total cases tested. Five cases are triple negative for EGFR, KRAS and BRAF alterations. The results from our study indicate that high EGFR copy number with concurrent KRAS or BRAF mutations is quite common in colon cancer and the therapeutic response with anti-EGFR agents in this patient population requires further investigation.
\end{abstract}

\section{Key words}

EGFR, KRAS, BRAF, Colorectal carcinoma, Targeted therapy

\section{I ntroduction}

Anti-EGFR targeted therapy of metastatic colorectal adenocarcinoma with monoclonal antibody cetuximab or panitumumab has been approved by FDA and become one of the standard treatments for patients with advanced colorectal adenocarcinoma ${ }^{[1]}$. Alterations of KRAS, EGFR and BRAF genes have shown significant impact on the therapeutic response to anti-EGFR treatment and overall survival ${ }^{[1-4]}$. Patients with wild-type KRAS and BRAF, or high EGFR copy number demonstrate better response to anti-EGFR therapy ${ }^{[1-4]}$. Correlation of these genetic alterations is not only 
important to the understanding of the mechanism of cancer development, but also key to patient management including selection of molecular tests and therapeutic agents. Currently, there is limited study on patients with both KRAS mutation and high EGFR copy number, which have opposite effects on anti-EGFR therapy. The prevalence of such "conflict" combination of genetic alterations in colon cancer patients has not been defined. The therapeutic response of such patients to cetuximab or panitumumab is unclear. To address some of these questions, we investigated the genetic-alteration profiles of KRAS, EGFR and BRAF and their correlation in 28 cases of colorectal adenocarcinoma.

\section{Materials and methods}

\subsection{Cases}

Twenty-eight cases of paraffin-embedded, formalin-fixed colon adenocarcinoma are randomly selected from the archives (2003-2009) of the Department of Pathology, SUNY Upstate Medical University. These cases include 4 well-differentiated, 16 moderately-differentiated and 8 poorly-differentiated adenocarcinomas. Four of them are metastasis to either lung or liver.

\subsection{Fluorescence in situ hybridization ( FISH)}

Gene copy number per cell is investigated by FISH using the LSI EGFR SpectrumOrange/CEP 7 SpectrumGreen probe (Vysis Inc., Abbott Laboratories, IL) as previously described ${ }^{[5]}$. FISH analysis is performed in a blinded fashion without knowledge of the patients' clinical characteristics and other genetic alterations. FISH positivity is defined as having polysomy of $\geqslant 3$ EGFR/CEP7 copies on average or EGFR amplification with $\geqslant 2$ ratio of EGFR to CEP 7 signals.

\subsection{DNA isolation for PCR}

Paraffin embedded tissue blocks with tumor are selected after reviewing the hematoxylin and eosin (HE)-stained slides. The tumor areas are encircled with a fine-tipped pen on the corresponding unstained slides. The encircled areas are scraped off with a syringe needle for DNA isolation. DNA is isolated using Zymo Pinpoint DNA isolation Kit (Zymo Research Corp., Orange, CA) according to the manufacturer's guidelines.

\subsection{Allele Specific PCR for BRAF mutation}

The extracted DNA is amplified by allele specific PCR with primer sets for BRAF V600E mutation, as previously described ${ }^{[6]}$. The primer sets included two forward primers with one covering the wild-type allele and the other covering the mutant allele. The two forward primers differ only in their 3' nucleotide sequences. One reverse primer is used with both forward primers. PCR is performed in a $50 \mu \mathrm{l}$ of reaction mixture: $1 \mu \mathrm{l}$ extracted DNA, $25 \mu \mathrm{l}$ HotStar Taq Master Mix (Qiagen, Valencia, CA), $0.6 \mu \mathrm{M}$ primers (Integrated DNA Technologies, Coralville, IA), and $24 \mu \mathrm{l} \mathrm{H}_{2} \mathrm{O}$ (Sigma-Aldrich, St. Louis, MO). The reaction mix is subjected to an initial activation step at $95^{\circ} \mathrm{C}$ for 15 minutes, 40 cycles of denaturation at $95^{\circ} \mathrm{C}$ for 5 seconds, annealing at $66^{\circ} \mathrm{C}$ for 5 seconds, extension at $72^{\circ} \mathrm{C}$ for 6 seconds, and a final extension step at $72^{\circ} \mathrm{C}$ for 10 minutes on the GeneAMP PCR system 9700 (PE Applied Biosystems, Foster City, CA). Water is used as negative control. These PCR products are subjected to electrophoresis on Ready Gel ${ }^{\circledR}$ Precast $10 \%$ TBE polyacrylamide gels (Bio-Rad, Hercules, CA). The gels are stained with ethidium bromide, visualized and photographed under UV light. Bands for both mutated and wild-type alleles are expected to be 149bp.

\subsection{Co-amplification at lower denaturation temperature-PCR (COLD-PCR) and DNA sequencing for KRAS mutation}

The primers target a 98bp amplicon in exon 2 of KRAS gene, covering all possible mutations in codons 12 [GGT] and 13 [GGC]. PCR pimers and running condition are adapted from previous publication ${ }^{[7]}$. 
The amplicon is directly sequenced with the same primers as above using the BigDye Terminator v1.1 cycle sequencing kit (Applied Biosystems) and analyzed with capillary electrophoresis on ABI PRISM 310 Genetic Analyzer.

\section{Results}

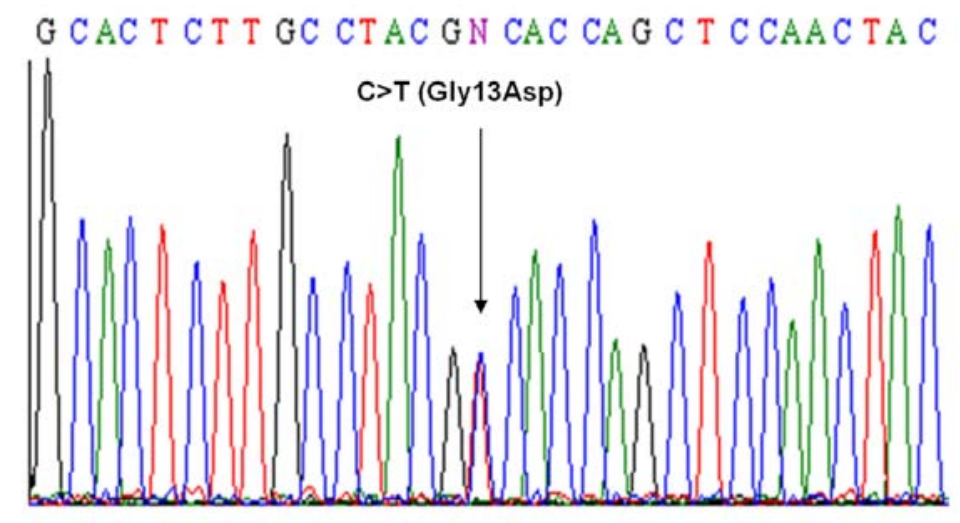

Figure 1. Example (Case 11) of KRAS gene mutation by COLD-PCR and DNA sequencing

High EGFR copy number (Figure 2), KRAS mutations (Figure 1) and BRAF mutations are found in 15 (54\%), 13 (46\%) and 3 (11\%) samples, respectively. All the cases of high EGFR copy number are associated with EGFR/CEP7 polysomy instead of EGFR amplification. Sixteen samples (13 KRAS-positive and 3 BRAF-positive) show mutual exclusion of KRAS and BRAF mutations (Figure 3). However, 6 (46\%) of the 13 KRAS-positive and 2 (66\%) of the 3 BRAF-positive samples have co-existing high EGFR copy number. Seven samples with high EGFR copy number are negative for both KRAS and BRAF mutations. Five cases are triple negative for EGFR high-copies, KRAS and BRAF mutations.
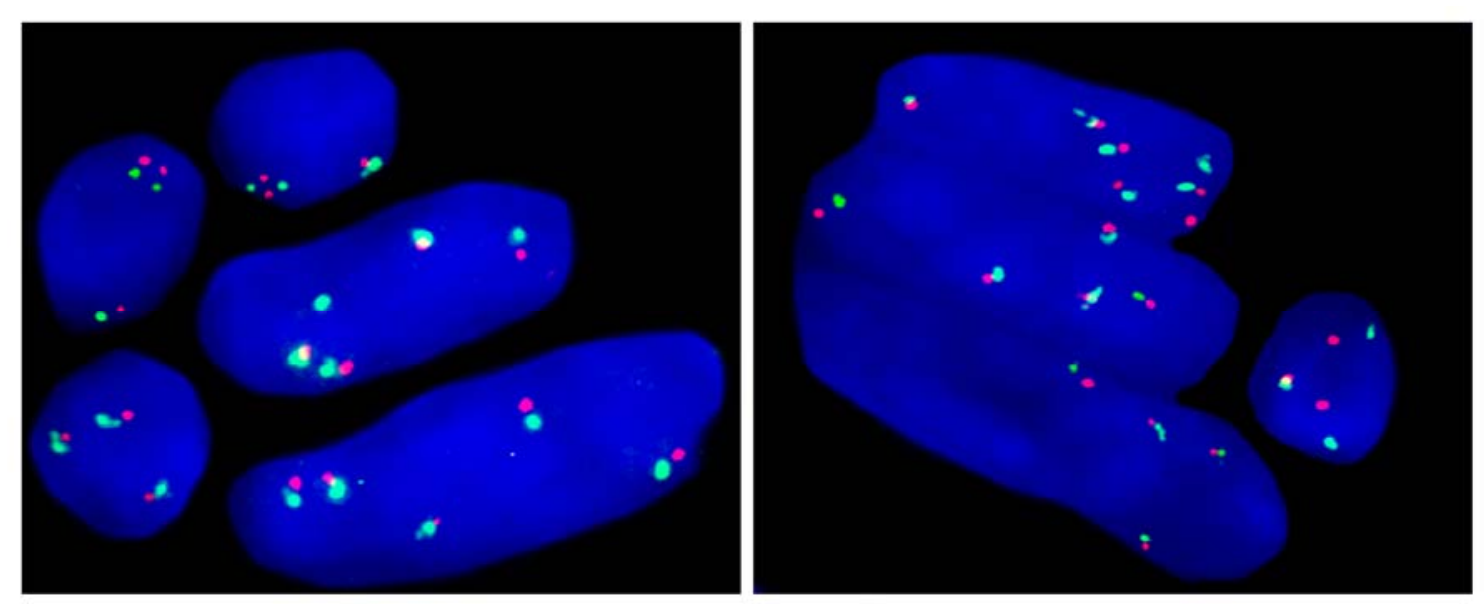

Figure 2. Examples of high EGFR copy number in colon adenocarcinoma (case 6 and 12) showing polysomy with increased EGFR (red) and CEP (green) signals (normal cells have 2 red and 2 green signals)

Additional base peak of $T$ is present at codon 13 of KRAS gene ( $C>T$, antisense), which renders G13D of amino acid change. 


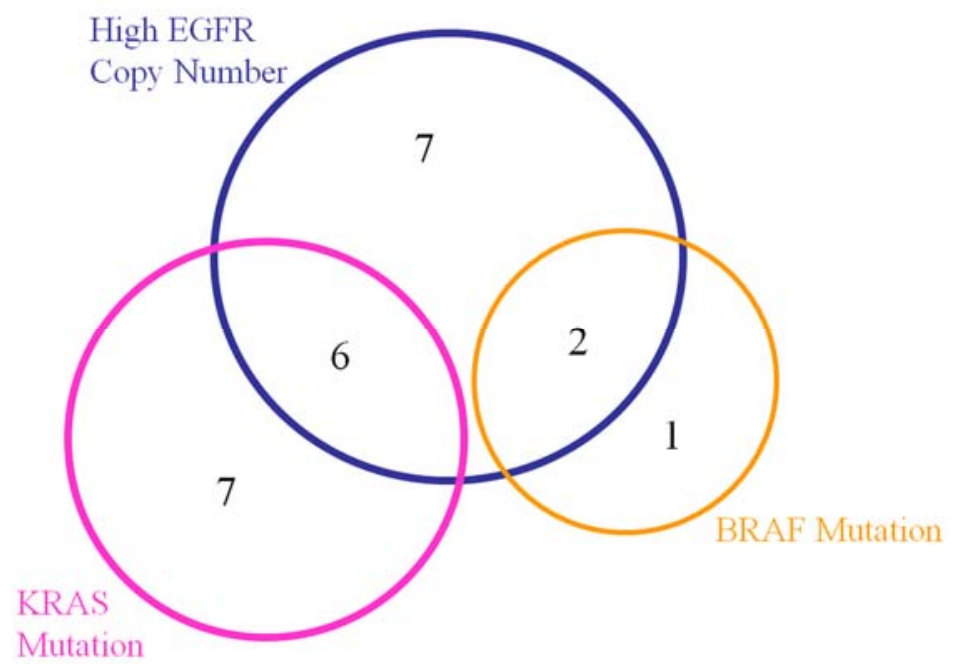

Figure 3. A diagram for correlation between KRAS, EGFR and BRAF genetic alterations. KRAS and BRAF mutations show mutually exclusive pattern. High EGFR copies shows co-existence with KRAS and BRAF mutations

\section{Discussion}

Although the colon cancers with KRAS mutation are lack of response to anti-EGFR therapy with Cetuximab or Panitumumab, approximately 50\% of the tumors without KRAS mutation (wild type) fail to respond to the treatment as well ${ }^{[8]}$. Therefore, it is imperative to reveal other biomarkers and their correlations for more accurate identification of appropriate patients for the targeted therapy. BRAF mutation in colon cancer has been known as a strong negative prognostic factor, and is being recognized as a predictive marker for response to anti-EGFR therapy as well ${ }^{[8,9]}$. In Laurent-Puig's study ${ }^{[8]}$, BRAF mutation was associated with not only lack of response, but also shorter progression-free survival (PFS) and overall survival (OS), in a pattern similar to the patients with KRAS mutation. In addition, among the tumors with wild type KRAS, EGFR FISH-positive tumors (polysomy) were associated with significantly higher response rate (RR, 71\%) than that with normal EGFR copy number (37\%), and with a trend toward longer PFS and OS ${ }^{[8]}$. Obviously, it is important to study the correlations among EGFR copy number, KRAS and BRAF mutations for setting up a reasonable assay's algorithm.

In lung adenocarcinoma, EGFR gene mutations are mutually exclusive with KRAS or BRAF gene mutations ${ }^{[10,11]}$. Tumor samples with mutation in one of these genes are unlikely to harbor mutations in the other genes, making it unnecessary to detect KRAS or BRAF gene mutations in EGFR positive cases. So far, EGFR gene mutation has not been identified in colon cancer. Instead, high EGFR gene copy number, either gene amplification or polysomy, are present in a subpopulation of colon cancer patients and has been used as a molecular marker for the selection of anti-EGFR therapy with either cetuximab or panitumumab ${ }^{[4,12]}$. As seen in lung cancer, KRAS and BRAF mutations are mutually exclusive in colon adenocarcinoma as well. However, co-existence of KRAS or BRAF mutation with EGFR high-copies is common in colon cancer, accounting for $46 \%$ of the KRAS positive cases and $66 \%$ of the BRAF positive cases respectively. Based on previous observation, there were almost no any colon cancers with KRAS mutation demonstrating benefit from anti-EGFR therapy ${ }^{[1,2]}$, which should include those with high copy EGFR. However, in those with wide type KRAS, the tumors with high copy EGFR have showed significantly better response to anti-EGFR therapy ${ }^{[8]}$. Since BRAF mutations are associated with resistance to anti-EGFR therapy as well ${ }^{[8,13,14]}$, it is unlikely that the tumors with concurrent EGFR high copies and BRAF mutations would get benefit from the treatment. Because around one third of colon adenocarcinomas harbor co-existing EGFR high-copies and KRAS or BRAF mutations observed in our study, test of only EGFR copy number for selecting patient is insufficient and not recommended. 
According our study, we suggest the following test algorithm: starting with KRAS mutation test, if it is negative, followed by BRAF mutation test. If BRAF mutation is again negative, EGFR copy number can be done if it is desired. Based on current data of clinical trials ${ }^{[8,9,15]}$, the degrees of benefit of anti-EGFR therapy are related the status of genetic alterations and are presumed as following: (EGFR high copy/KRAS wild-type/BRAF wild-type) > (EGFR normal copy/KRAS wild-type/BRAF wild-type) $>$ (BRAF mutant) $>$ (KRAS mutant). Apparently, detection of multiple relevant genetic alterations in colon cancer would help to identify more appropriate candidates for the anti-EGFR therapy with Cetuximab or Panitumumab.

\section{References}

[1] Allegra CJ, Jessup JM, Somerfield MR, et al. Society of clinical oncology provisional clinical opinion: testing for KRAS gene mutations in patients with metastatic colorectal carcinoma to predict response to anti-epidermal growth factor receptor monoclonal antibody therapy. J Clin Oncol. 2009; 27: 2091-2096. PMid: 19188670 http://dx.doi.org/10.1200/JCO.2009.21.9170

[2] Siena S, Sartore-Bianchi A , Nicolantonio FD, et al. Biomarkers Predicting Clinical Outcome of Epidermal Growth Factor Receptor - Targeted Therapy in Metastatic Colorectal Cancer. J Natl Cancer Inst. 2009; 101: 1308-1324. PMid: 19738166

[3] Mao C, Liao RY, Qiu LX, et al. BRAF V600E mutation and resistance to anti-EGFR monoclonal antibodies in patients with metastatic colorectal cancer: a meta-analysis. Mol Bio Rep. 2011; 38: 2219-2223. PMid: 20857202

[4] Moroni M, Veronese S and Benvenuti S et al. Gene copy number for epidermal growth factor receptor (EGFR) and clinical response to antiEGFR treatment in colorectal cancer: a cohort study. Lancet Oncol. 2005; 6: 279-86. PMid: 15863375

[5] El-Zammar OA, Zhang S and Katzenstein ALA. Comparison of FISH, PCR, and immunohistochemistry in assessing EGFR Status in lung adenocarcinoma and correlation with clinicopathologic features. Diagn Mol Pathol. 2009; 18: 133-137. PMid: 19704257

[6] Jin L, Sebo TJ, Nakamura N, et al. BRAF mutation analysis in fine needle aspiration (FNA) cytology of the thyroid. Diagn Mol Pathol. 2006; 15: 136-143. PMid:16932068

[7] Zuo Z, Chen SS, Chandra PK, et al. Application of COLD-PCR for improved detection of KRAS mutations in clinical samples. Mod Pathol. 2009; 22: 1023-1031. PMid:19430420

[8] Laurent-Puig P, Cayre Anne, Manceau G, et al. Analysis of PTEN, BRAF, and EGFR status in determining benefit from Cetuximab therapy in wild-type KRAS metastatic colon cancer. J Clin Oncol. 2009; 27: 5924-5930. http://dx.doi.org/10.1200/JCO.2008.21.6796

[9] Yokota T. Are KRAS/BRAF mutations potent prognostic and/or predictive biomarkers in colorectal cancers? Anticancer Agents Med Chem. 2012; 12: 163-171. PMid: 22043994

[10] Schmid K, Oehl N, Wrba F, et al. EGFR/KRAS/BRAF mutations in primary lung adenocarcinomas and corresponding locoregional lymph node metastases. Clin Cancer Res. 2009; 15: 4554-4560. PMid:19584155

[11] Borràs E, Jurado I, Hernan I, et al. Clinical pharmacogenomic testing of KRAS, BRAF and EGFR mutations by high resolution melting analysis and ultra-deep pyrosequencing. BMC Cancer. 2011; 11: 406. PMid: 21943394. http://dx.doi.org/10.1186/1471-2407-11-406

[12] Cappuzzo F, Finocchiaro G, Rossi E, et al. EGFR FISH assay predicts for response to cetuximab in chemotherapy refractory colorectal cancer patients. Ann Oncol. 2008; 19: 717-723. PMid: 17974556. http://dx.doi.org/10.1093/annonc/mdm492

[13] Di Nicolantonio F, Martini M, Molinari F et al. Wild-type BRAF is required for response to panitumumab or cetuximab in metastatic colorectal cancer. J Clin Oncol. 2008; 26: 5668-5670. PMid: 19001320

[14] Loupakis F, Ruzzo A, Cremolini C, et al. KRAS codon 61, 146 and BRAF mutations predict resistance to cetuximab plus irinotecan in KRAS codon 12 and 13 wild-type metastatic colorectal cancer. Br J Cancer. 2009; 101: 715-721. PMid:19603018

[15] Park JH, Han SW, Oh DY, et al. Analysis of KRAS, BRAF, PTEN, IGF1R, EGFR intron 1 CA status in both primary tumors and paired metastases in determining benefit from cetuximab therapy in colon cancer. Cancer Chemother Pharmacol. 2011; 68: 1045-1055. PMid: 21340604 